\title{
High-velocity gas toward the LMC resides in the Milky Way halo
}

\author{
P. Richter ${ }^{1,2}$, K. S. de Boer ${ }^{3}$, K. Werner ${ }^{4}$, and T. Rauch ${ }^{4}$ \\ ${ }^{1}$ Institut für Physik und Astronomie, Universität Potsdam, Karl-Liebknecht-Str. 24/25, 14476 Golm, Germany \\ ${ }^{2}$ Leibniz-Institut für Astrophysik Potsdam (AIP), An der Sternwarte 16, 14482 Potsdam, Germany \\ e-mail: prichter@astro.physik.uni-potsdam.de \\ 3 Argelander-Institute for Astronomy, University of Bonn, Auf dem Hügel 71, 53121 Bonn, Germany \\ ${ }^{4}$ Institute for Astronomy and Astrophysics, Kepler Center for Astro and Particle Physics, Eberhard Karls Universität Tübingen, \\ Sand 1, 72076 Tübingen, Germany
}

Received 25 September 2015 / Accepted 3 November 2015

\begin{abstract}
Aims. To explore the origin of high-velocity gas in the direction of the Large Magellanic Cloud, (LMC) we analyze absorption lines in the ultraviolet spectrum of a Galactic halo star that is located in front of the LMC at $d=9.2_{-7.2}^{+4.1} \mathrm{kpc}$ distance.

Methods. We study the velocity-component structure of low and intermediate metal ions (C II, Si II, Si III) in the spectrum of RX J0439.8-6809, as obtained with the Cosmic Origins Spectrograph (COS) onboard the Hubble Space Telescope (HST), and measure equivalent widths and column densities for these ions. We supplement our COS data with a Far-Ultraviolet Spectroscopic Explorer (FUSE) spectrum of the nearby LMC star Sk -6959 and with H I $21 \mathrm{~cm}$ data from the Leiden-Argentina-Bonn (LAB) survey.

Results. Metal absorption toward RX J0439.8-6809 is unambiguously detected in three different velocity components near $v_{\text {LSR }}=0$, +60 , and $+150 \mathrm{~km} \mathrm{~s}^{-1}$. The presence of absorption proves that all three gas components are situated in front of the star, thus located in the disk and inner halo of the Milky Way. For the high-velocity cloud (HVC) at $v_{\mathrm{LSR}}=+150 \mathrm{~km} \mathrm{~s}^{-1}$, we derive an oxygen abundance of $[\mathrm{O} / \mathrm{H}]=-0.63(\sim 0.2$ solar) from the neighboring $\mathrm{Sk}-6959$ sight line, in accordance with previous abundance measurements for this HVC. From the observed kinematics we infer that the HVC hardly participates in the Galactic rotation.

Conclusions. Our study shows that the HVC toward the LMC represents a Milky Way halo cloud that traces low column density gas with relatively low metallicity. We rule out scenarios in which the HVC represents material close to the LMC that stems from a LMC outflow.
\end{abstract}

Key words. Galaxy: halo - Galaxy: evolution - ISM: abundances - ISM: structure

\section{Introduction}

The Milky Way is surrounded by large amounts of neutral and ionized gas that can be observed in absorption, against bright extragalactic background sources, or in emission (e.g., in H I $21 \mathrm{~cm}$ ). This gas is manifold in origin and chemical composition, reflecting the various processes that determine the gas distribution around Milky-Way-type galaxies as part of cosmological structure formation and galaxy evolution (e.g., gas infall, supernova-driven outflows, tidal interactions).

Extraplanar gas features in the Galactic halo manifest themselves in absorption and emission features at high radial velocities, which are incompatible with those expected from Galactic rotation models. Coherent gas streams with radial velocities $\left|v_{\text {LSR }}\right| \geq 100 \mathrm{~km} \mathrm{~s}^{-1}$ are usually referred to as high-velocity clouds (HVCs), while those with somewhat lower velocities $\left(\left|v_{\mathrm{LSR}}\right|=50-100 \mathrm{~km} \mathrm{~s}^{-1}\right)$ are called intermediate-velocity clouds (IVCs; see Wakker \& van Woerden 1998; Richter 2006; Putman et al. 2012, for recent reviews).

Previous studies have demonstrated that the majority of the large IVCs and HVCs are located in the inner Galactic halo at distances $d \leq 20 \mathrm{kpc}$ (e.g., Wakker et al. 2007, 2008; Thom et al. 2006, 2008). A prominent exception is the so-called Magellanic Stream, which represents a massive structure $\left(10^{8}-10^{9} M_{\odot}\right)$ of neutral and ionized gas at $d \sim 50 \mathrm{kpc}$ that originates from the interaction of the two Magellanic Clouds orbiting the Milky Way (e.g., Wannier \& Wrixon 1972; Fox et al. 2010, 2013, 2014; Richter et al. 2013).

Among the various HVCs, the high-velocity cloud near $+150 \mathrm{~km} \mathrm{~s}^{-1}$ in front of the LMC (hereafter referred to as HVCtwLMC) is among the best studied, owing to the fact that there are many bright LMC stars in the background that can be used to study the HVC with the absorption spectroscopy method. Previous observations indicate that HVCtwLMC is a relatively metal-poor ( $\alpha$ abundance: $\sim 0.3$ solar; Lehner et al. 2009 , hereafter referred to as L09), multiphase gas cloud that is predominantly ionized, but consists of considerable small-scale structure, denser subclumps, and dust (Richter et al. 1999; Welty et al. 1999; Smoker et al. 2015). Earlier studies of HVCtwLMC have favored a Galactic origin (e.g., Savage \& de Boer 1981; de Boer et al. 1990; Richter et al. 1999), and some later studies proposed that HVCtwLMC is located close to the LMC, representing metal-enriched gaseous material that stems from an outflow caused by the enhanced star formation activity in the Magellanic Clouds (Staveley-Smith et al. 2003; L09).

In this paper we demonstrate that HVCtwLMC is located in front of the star RX J0439.8-6809 (Fig. 1) at a distance of $d \leq 13.3 \mathrm{kpc}$ (Werner \& Rauch 2015), thus proving that the gas resides within the inner halo of the Milky Way and is not related to a supposed LMC outflow. The paper is organized as follows. In Sect. 2 we briefly describe the observations and data analysis. 


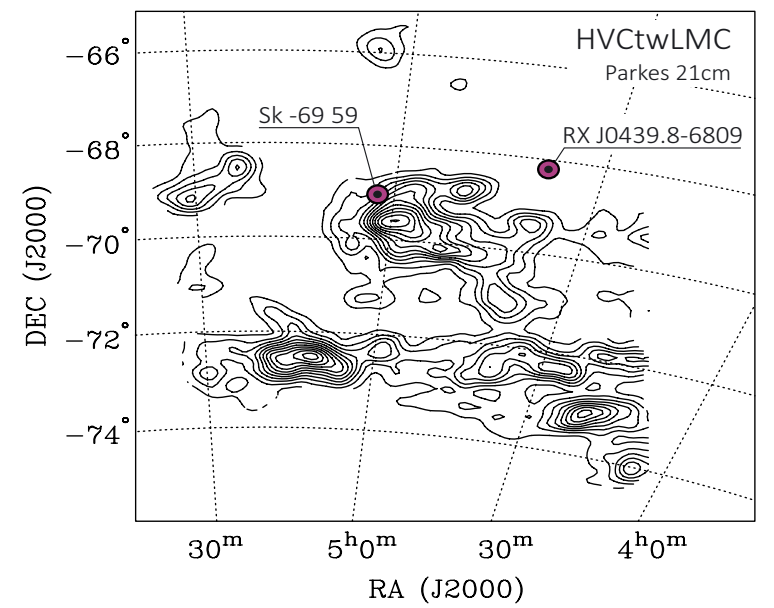

Fig. 1. Sky position of the two background stars RX J0439.8-6809 (at $d<13.3 \mathrm{kpc}$ ) and $\mathrm{Sk}-6959$ (at $d>45 \mathrm{kpc}$ ). The contours show the H I column density in the velocity range $v_{\mathrm{LSR}}=100-165 \mathrm{~km} \mathrm{~s}^{-1}$ in steps of $2 \times 10^{18} \mathrm{~cm}^{-2}$ starting at $2 \times 10^{18} \mathrm{~cm}^{-2}$, as observed in the $21 \mathrm{~cm}$ Parkes multibeam survey of the LMC (adapted from Staveley-Smith et al. 2003). The HVC presumably is much more extended in ionized gas, as indicated by the detection of UV absorption outside the $21 \mathrm{~cm}$ boundaries (see also L09).

In Sect. 3 we present the results from the spectral analysis. In Sect. 4 we explore the space motion of HVCtwLMC, discuss its chemical composition, and indicate possible origins of the gas. We conclude our study in Sect. 5 .

\section{Observations and data handling}

For our experiment we make use of archival ultraviolet (UV) spectral data of the extremely hot white dwarf (WD) RX J0439.8-6809. Since its discovery in 1994 as ROSAT supersoft X-ray source, RX J0439.8-6809 was regarded as a WD located in the LMC (Greiner et al. 1994). A recent spectral analysis of RX J0439.8-6809 by Werner \& Rauch (2015) based on COS data reveals, however, that the star has a spectroscopic distance of just $d=9.2_{-7.2}^{+4.1}$, thus located within the Milky Way halo $\sim 5.6 \mathrm{kpc}$ below the Galactic disk. The distance and the position (at $l=279.9, b=-37.1$ ) in front of the LMC make RX J0439.8-6809 the ideal target to constrain the distance of intermediate- and high-velocity gas in the direction of the Magellanic Clouds.

We use the same COS data set as presented in Werner \& Rauch (2015). RX J0439.8-6809 was observed with HST/COS using the G130M grating that covers the wavelength range between $\lambda=1150-1450 \AA$ at a spectral resolution of $R \approx$ 15 000-20000 (FWHM 15-20 $\mathrm{km} \mathrm{s}^{-1}$ ). The total exposure time was $14080 \mathrm{~s}$. We retrieved the original $\mathrm{x} 1 \mathrm{~d}$ fits files (processed with the CALCOS pipeline v2.17.3) from the MAST archive and coadded the individual sciences exposures following the procedure described in Richter et al. (2015). The signal-to-noise $(\mathrm{S} / \mathrm{N})$ in the final coadded spectrum is $\sim 15$ per resolution element at $1300 \AA$.

We supplement our COS data with a FUSE far-UV spectrum of the LMC Wolf-Rayet star Sk-6959, located at $l=280.1$, $b=-34.8$, thus $\sim 2.5 \mathrm{deg}$ away from RX J0439.8-6809. The motivation for including these FUSE data is to have a reference spectrum of a nearby line of sight that displays all of the velocity components of gas in the general direction of the LMC at high $\mathrm{S} / \mathrm{N}$ (including gas within the LMC). In addition, FUSE provides access to important interstellar lines (e.g., from O I and Fe II) that we later use to explore the chemical composition of the HVC.

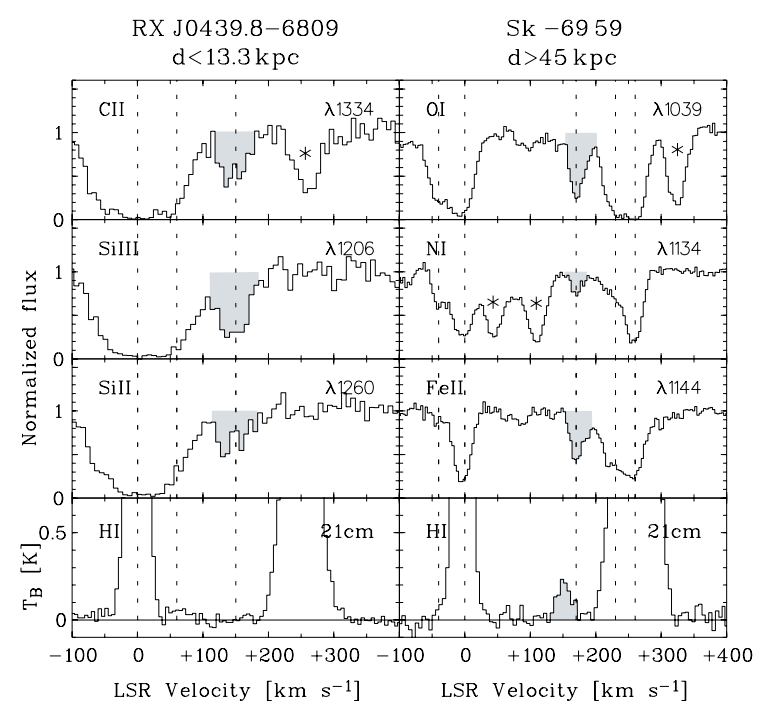

Fig. 2. Absorption and emission profiles of different ions toward RX J0439.8-6809 and Sk-6959. HVC absorption/emission features are indicated with the gray-shaded area. The dotted lines indicate the LSR velocities of main absorption components in the Milky Way disk/halo and in the LMC. The star symbol indicates absorption from blending interstellar lines.

The FUSE data were also retrieved from the MAST archive and were reduced in a way similar as described in Richter et al. (2013).

The sky positions of RX J0439.8-6809 and Sk-6959 are plotted in Fig. 1 together with an $\mathrm{HI} 21 \mathrm{~cm}$ map of the HVCtwLMC (from Staveley-Smith et al. 2003). For the spectral analysis of the COS and FUSE data, we used the customwritten span software package, which is based on ESO-MIDAS and its fitlyman spectral library (Fontana \& Ballester 1995). Equivalent widths and column densities were derived from a direct pixel integration of the absorption profiles using the apparent-optical depth (AOD) method (Savage \& Sembach 1991). Atomic data have been adopted from the compilation by Morton (2003).

To derive the H I column densities (or limits) in the direction of the two background sources, we consider publicly available $21 \mathrm{~cm}$ spectral data from the LAB survey (Kalberla et al. $2005)$ and the relation $N(\mathrm{HI})=1.823 \times 10^{18} \mathrm{~cm}^{-2} \int_{v_{\min }}^{v_{\max }} T_{\mathrm{B}} \mathrm{d} v$.

\section{Results from the spectral analysis}

In the left panel of Fig. 2, we show the (interstellar) absorption profiles of C II $\lambda 1334.53$, Si III $\lambda 1206.50$, and Si II $\lambda 1260.42$ in the COS spectrum of RX J0439.8-6809 plotted on a local standard of rest (LSR) velocity scale with the LAB $21 \mathrm{~cm}$ emission profile. Absorption in these sight lines is detected in three main absorption components centered at $v_{\mathrm{LSR}}=0,+60$ and $+150 \mathrm{~km} \mathrm{~s}^{-1}$ (dotted lines). The intermediate-velocity component at $v_{\mathrm{LSR}}=+60 \mathrm{~km} \mathrm{~s}^{-1}$ blends with the very strong local disk absorption at zero velocities, as observed along many LMC sight lines (see Danforth et al. 2002). The high-velocity gas at $v_{\text {LSR }}=+150 \mathrm{~km} \mathrm{~s}^{-1}$ is well separated from the other velocity components. H I $21 \mathrm{~cm}$ emission is only seen near zero velocities (Galactic disk) and near $v_{\mathrm{LSR}}=+250 \mathrm{~km} \mathrm{~s}^{-1}$, the latter component coming from neutral gas in the LMC behind RX J0439.8-6809, i.e., gas not present in absorption. The lack of $21 \mathrm{~cm}$ emission at $+150 \mathrm{~km} \mathrm{~s}^{-1}$ indicates that the column density of H I in HVCtwLMC toward RX J0439.8-6809 is less than the detection limit (see Table 1; Fig. 1). 
Table 1. HVC absorption- and emission-line measurements.

\begin{tabular}{lrrrr}
\hline \hline Ion & $\lambda_{0}[\AA]$ & Instrument & \multicolumn{1}{c}{$W_{\lambda}[\mathrm{m} \AA]$} & $\log N$ \\
\hline \multicolumn{5}{c}{ RX J0439.8-6809; $v_{\text {LSR }}=+150 \mathrm{~km} \mathrm{~s}^{-1}$} \\
\hline C II & 1334.53 & COS & $116 \pm 12$ & $>13.90$ \\
Si III & 1206.50 & COS & $177 \pm 12$ & $>13.14$ \\
Si II & 1260.42 & COS & $98 \pm 11$ & $>12.89$ \\
Si II & 1193.29 & COS & $78 \pm 11$ & $13.10 \pm 0.12$ \\
Si II & 1190.42 & COS & $10 \pm 4$ & $13.04 \pm 0.16$ \\
H I & $21 \mathrm{~cm}$ & LAB & & $<18.61$ \\
\hline \multicolumn{5}{c}{ Sk-6959; $v_{\text {LSR }}=+170 \mathrm{~km} \mathrm{~s}^{-1}$} \\
\hline O I & 1039.23 & FUSE & $78 \pm 6$ & $15.12 \pm 0.04$ \\
N I & 1134.98 & FUSE & $21 \pm 4$ & $13.68 \pm 0.07$ \\
N II & 1083.99 & FUSE & $156 \pm 7$ & $\geq 14.25$ \\
Fe II & 1144.94 & FUSE & $63 \pm 5$ & $13.83 \pm 0.05$ \\
P II & 1152.82 & FUSE & $\leq 12$ & $\leq 12.10$ \\
H I & $21 \mathrm{~cm}$ & LAB & & $19.06 \pm 0.06$ \\
\hline \multicolumn{5}{c}{}
\end{tabular}

In the right panel of Fig. 2 we show the absorption profiles of O I $\lambda 1039.23$, N I $\lambda 1134.98$, and Fe II $\lambda 1144.94$ in the FUSE spectrum of $\mathrm{Sk}-6959$. Here, the high-velocity component is shifted toward $v_{\text {LSR }}=+170 \mathrm{~km} \mathrm{~s}^{-1}$, while no intermediatevelocity component is present. Absorption at $v_{\mathrm{LSR}}=+200$ to $+270 \mathrm{~km} \mathrm{~s}^{-1}$ stems from gas within the LMC. In contrast to RX J0439.8-6809, weak $21 \mathrm{~cm}$ emission is detected in the HVC component toward Sk -6959 , but the emission is shifted to velocities near $v_{\mathrm{LSR}}=+150 \mathrm{~km} \mathrm{~s}^{-1}$. We attribute the velocity shift to beam-smearing effects in the LAB $21 \mathrm{~cm}$ data that have a spatial resolution of $\sim 36^{\prime}$.

For the HVCtwLMC gas, we have derived ion equivalent widths and column densities from the COS, FUSE, and LAB data. The results are summarized in Table 1.

\section{Properties of the high-velocity cloud}

\subsection{Location and kinematics}

With $l=279.9, b=-37.1$ and $d \leq 13.3 \mathrm{kpc}$, the HVC's vertical distance to the disk is limited to $z \leq 8.0 \mathrm{kpc}$. The observed radial velocity, however, does not constrain the space velocity of the gas. To evaluate the space motion of HVCtwLMC, we use the geometry of the sight line to RX J0439.8-6809 as given in Fig. 3.

First, we consider velocities in the Galactic standard of rest (GSR), i.e., velocities with respect to the location of the Sun without the Galactic rotation. Assuming that the circular orbit speed of the Sun is $v_{\text {rot }}=255 \mathrm{~km} \mathrm{~s}^{-1}$ (Reid et al. 2014), the component of the velocity of the Sun along the line of sight to the star is $\sin (l) \cos (b) v_{\text {rot }}=201 \mathrm{~km} \mathrm{~s}^{-1}$ (see Fig. 3, panel c). The observed radial velocity of HVCtwLMC is $+150 \mathrm{~km} \mathrm{~s}^{-1}$, so this gas has $+51 \mathrm{~km} \mathrm{~s}^{-1}$ in the GSR system in this direction (Fig. 3, panelc).

To further constrain the space motion of HVCtwLMC, we decompose the HVC space velocity vector into three orthogonal components: $v_{\text {rot }}, v_{\text {axis }}$, and $v_{z}$ (see Fig. 3, panel b; Kaelble et al. 1985; de Boer 2005). Because of the position of HVCtwLMC with respect to the Sun, its $v_{\text {axis }}$ component, which is almost perpendicular to the sight line, cannot contribute significantly to the observed $v_{\text {rad }}$. A radial velocity of $v_{\text {rad }}(\mathrm{GSR})=+51 \mathrm{~km} \mathrm{~s}^{-1}$ in this direction can then be understood by either having $v_{\text {rot }}=$ $64 \mathrm{~km} \mathrm{~s}^{-1}$ or by having $v_{z}=-85 \mathrm{~km} \mathrm{~s}^{-1}$. This is because the velocity components $v_{z}$ and $v_{\text {rot }}$ of HVCtwLMC in the discussed geometry would have to combine to result in $51 \mathrm{~km} \mathrm{~s}^{-1} \simeq$ $\cos (b) v_{\text {rot }}-\sin (b) v_{z}$, where $v_{z}$ has a negative sign for gas falling toward the disk.

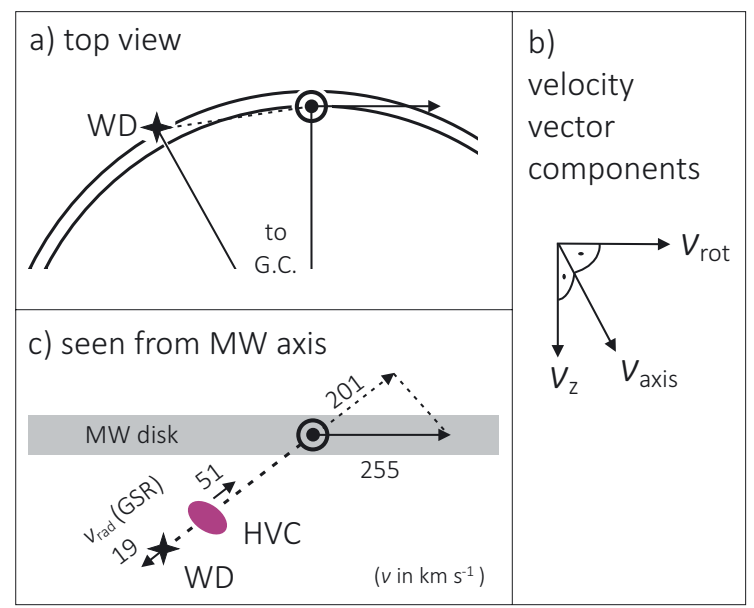

Fig. 3. Geometry of Sun and RX J0439.8-6809 in the Galaxy shown schematically. Panel a): view from above the Milky Way plane. Panel b): decomposition of the space velocity in the Milky Way into the components $v_{\text {rot }}$, the velocity parallel to the disk in the direction of circular rotation, $v_{\text {axis }}$, the velocity component pointing (parallel to the disk) at the rotation axis of the Galaxy, and $v_{z}$, the velocity perpendicular to the disk; the outward direction has a positive sign. Panel c): the geometry as seen from the Milky Way rotation axis. Velocities are given in GSR (see Sect. 4.1). The radial velocity of the star is $+220 \mathrm{~km} \mathrm{~s}^{-1}$ (Werner \& Rauch 2015).

Since essentially all neutral HVC gas in the halo is found to be moving toward the MW disk (e.g., Schwarz \& de Boer 2004), we conclude that the radial velocity of HVCtwLMC in the GSR system is mostly due to the vertical motion of the gas $\left(v_{z}\right)$ with respect to the plane, while the contribution of the rotation $\left(v_{\text {rot }}\right)$ must be small. In other words, HVCtwLMC is hardly participating in the Galactic rotation. Because $v_{\text {axis }}$ is, as said above, unknown, we can only infer these limits to the space motion of HVCtwLMC. For further details on this type of HVC velocity analysis, see de Boer \& Savage (1983, 1984).

\subsection{Metallicity and ionization conditions}

The simultaneous presence of Si II and Si III toward RX J0439.8-6809 provides strong evidence for the presence of ionized gas in HVCtwLMC. As discussed in Richter et al. (2015), it is likely that in diffuse circumgalactic environments these two ionization stages of $\mathrm{Si}$ trace predominantly complementary gas phases. The similar column densities of Si II and Si III (Table 1) then indicate that there is at least as much ionized hydrogen in HVCtwLMC toward RX J0439.8-6809 as neutral hydrogen. The column density of Si II suggests that the amount of neutral hydrogen is clearly below the upper limit as derived from H I $21 \mathrm{~cm}$ (see Table 1). Alternatively, if the gas were to have solar composition, Si would be depleted into dust grains, but by at most 1 dex.

For other abundance ratios, we refer to data from the nearby sight line to Sk -6959 (separated by $\sim 2.5 \mathrm{deg}$ ) because for the sight line toward RX J0439.8-6809 no H I $21 \mathrm{~cm}$ emission is detected in the LAB data and OI $\lambda 1302.17$ absorption in the COS spectrum is contaminated by strong airglow lines. The most useful ion ratio to determine the overall metallicity of the gas is the $\mathrm{O} / \mathrm{HI}$ ratio. Neutral oxygen and neutral hydrogen have almost identical ionization potentials and in neutral gas both species are coupled through a strong charge-exchange reaction. In addition, the $\alpha$ element oxygen is not significantly depleted into dust grains (e.g., Savage \& Sembach 1996). We use H I $21 \mathrm{~cm}$ and O I $\lambda 1039.23$ toward Sk-6959 (see Fig. 2) 
to derive the metallicity of HVCtwLMC. With the column densities given in Table 1 and solar reference abundances from Asplund et al. (2009), we determine a metallicity/alpha abundance of $[\mathrm{O} / \mathrm{H}]=-0.63 \pm 0.05(\sim 0.23$ solar $)$, which is in good agreement with previous $[\mathrm{O} / \mathrm{H}]$ estimates for the $\mathrm{HVC}$ in this direction (L09).

Another valuable indicator for the enrichment history of the gas is the nitrogen abundance. From the N I column density, we infer $[\mathrm{N} / \mathrm{H}]=-1.21 \pm 0.08$, which is substantially lower than $[\mathrm{O} / \mathrm{H}]$. One reason for a low $\mathrm{N} / \mathrm{O}$ ratio may be $\mathrm{SN}$ type II-dominated enrichment, as observed in other prominent HVCs (e.g., Richter et al. 2001, 2013) and in many extragalactic absorption systems (e.g., Pettini et al. 2008). However, an alternative and similarly plausible explanation is that the low N I column density is a result of ionization effects that become important for neutral nitrogen in low-density environments (e.g., Fox et al. 2013, their Fig. 10). The latter conclusion is supported by the shape of the N II $\lambda 1083.99$ line, which exhibits strong absorption at $+170 \mathrm{~km} \mathrm{~s}^{-1}$. Because of line saturation, we can only give a lower limit of $\log N(\mathrm{~N}$ II $)=14.25$, which does not help to further pinpoint the intrinsic N/O ratio in HVCtwLMC without knowing the exact ionization conditions. Similar arguments hold for iron, for which we determine an apparent abundance of $[\mathrm{Fe} / \mathrm{H}]=-0.73 \pm 0.06$, thus being close to the derived $[\mathrm{O} / \mathrm{H}] \mathrm{ra}-$ tio. In predominantly neutral gas environments such a small difference between $[\mathrm{O} / \mathrm{H}]$ and $[\mathrm{Fe} / \mathrm{H}]$ would indicate very little dust depletion in the HVC in this direction. In HVCtwLMC, however, the observed $\mathrm{Fe}$ II/H I ratio more likely reflects a considerable amount of $\mathrm{H}$ II that coexists with singly-ionized iron in mostly ionized gas regions (see also L09), so that an intrinsically lower $[\mathrm{Fe} / \mathrm{H}]$ due to dust depletion or nucleosynthetic effects cannot be ruled out.

In summary, the analysis of $[\mathrm{N} / \mathrm{O}]$ and $[\mathrm{Fe} / \mathrm{H}]$ remains inconclusive with respect to the enrichment history and dust properties of the gas because of the unknown ionization conditions. The relatively low oxygen abundance of HVCtwLMC is in line with an extragalactic origin of the gas, similar as for other HVCs such as Complex C and Complex A (Wakker et al. 1999; Richter et al. 2001). The gas could also have originated in the Galactic halo, as gas shed by metal-poor halo stars during their red-giant phase (de Boer 2004) or from metal-poor gas in the outer Milky Way disk. That the gas would be some form of outflow from the LMC is no longer tenable because of the above derived distance limit.

\subsection{Total mass}

To provide an approximate mass limit of HVCtwLMC, we use the absorption statistics on O I presented in L09. We further assume that the distance of the cloud is $d=5 \mathrm{kpc}$ and the angular size is $A=100 \mathrm{deg}^{2}$, which is the angular size of the LMC. With these numbers, the physical size of the cloud is only $0.76 \mathrm{kpc}^{2}$ and the neutral gas mass is small, $M_{\mathrm{HVC}} \approx 4 \times 10^{4} M_{\odot}$. Depending on the ionization fraction of the gas and the spatial extent of the HVC beyond the LMC boundaries, the total gas mass might be substantially higher than this estimate. Additional absorption-line observations of QSOs that are located around the LMC would be very helpful to better constrain the size and mass of HVCtwLMC.

\section{Conclusions}

Our observations demonstrate that the HVC in the direction of the LMC (as well as all other absorption components) are located in front of the star RX J0439.8-6809 at $<13.3 \mathrm{kpc}$ distance. They all are part of the Galaxy and do not belong to some LMC outflow. Thus, HVCtwLMC is in line with other prominent HVCs for which reliable distance estimates exist and that are located within $20 \mathrm{kpc}$ distance, such as Complex A, Complex C, the Cohen Stream, and the Smith Cloud (van Woerden et al. 1999; Wakker et al. 2007, 2008; Thom et al. 2006, 2008). The derived oxygen abundance of HVCtwLMC ( $\sim 0.23$ solar) does not allow us to draw firm conclusions about its origin; both scenarios, a Galactic and an extragalactic origin, are in line with the observations.

The results presented here once again underline the importance of distance determinations of HVCs for our understanding of the spatial distribution of gas in the Milky Way halo.

\section{References}

Asplund, M., Grevesse, N., Jacques Sauval, A., \& Scott, P. 2009, ARA\&A, 47, 481

Danforth, C. W., Howk, J. C., Fullerton, A. W., Blair, W. P., \& Sembach, K. R. 2002, ApJS, 139, 81

de Boer, K. S. 2004, A\&A, 419, 527

de Boer, K. S. 2005, in Extra-planar Gas, ed. R. Braun, ASP Conf. Ser., 331, 49 de Boer, K. S., \& Savage, B. D. 1983, A\&A, 265, 210

de Boer, K. S., \& Savage, B. D. 1984, A\&A, 136, L7

de Boer, K. S., Morras, R., \& Bajaja, E. 1990, A\&A, 233, 523

Fontana, A., \& Ballester, P. 1995, ESO Messenger, 80, 37

Fox, A. J., Wakker, B. P., Smoker, J. V., et al. 2010, ApJ, 718, 1046

Fox, A. J., Richter, P., Wakker, B. P., et al. 2013, ApJ, 772, 110

Fox, A. J., Wakker, B. P., Barger, A., et al. 2014, ApJ, 787, 147

Greiner, J., Hasinger, G., \& Thomas, H.-C. 1994, A\&A, 281, L61

Kaelble, A.,de Boer, K. S., \& Grewing, M. 1985, A\&A, 143, 408

Kalberla, P. M. W., Burton, W. B., Hartmann, D., et al. 2005, A\&A, 440, 775

Lehner, N., Staveley-Smith, L., \& Howk, J. C. 2009, ApJ, 702, 940

Morton, D. C. 2003, ApJS, 149, 205

Pettini, M., Zych., B. J., Steidel, C. C., \& Chaffee, F. H. 2008, MNRAS, 385, 2011

Putman, M. E., Peek, J. E. G., \& Joung, M. R. 2012, ARA\&A, 50, 491

Reid, M. J., Menten, K. M., Brunthaler, A., et al. 2014, ApJ, 783, 130

Richter, P. 2006, Rev. Mod. Astron., 19, 31

Richter, P., de Boer, K. S., Bomans, D. J., et al. 1999, Nature, 402, 386

Richter, P., Sembach,K. R., Wakker, B. P., et al. 2001, ApJ, 559, 318

Richter, P., Fox, A. J., Wakker, B. P., et al. 2013, ApJ, 772, 111

Richter, P., Wakker, B. P., Fechner, C., et al. 2015, A\&A, submitted

Savage, B. D., \& de Boer, K. S. 1981, ApJ, 243, 460

Savage, B. D., \& Sembach, K. R. 1991, ApJ, 379, 245

Savage, B. D., \& Sembach, K. R. 1996, ARA\&A, 34, 279

Schwarz, U. J., \& de Boer, K. S. 2004, in High-Velocity Clouds, eds. H. van Woerden et al. (Kluwer Academic Publishers), 55

Smoker, J. V., Fox, A., \& Keenan, F. P. 2015, MNRAS, 451, 4346

Staveley-Smith, L., Kim, S., Calabretta, M. R., Haynes, R. F., \& Kesteven, M. J. 2003, MNRAS, 339, 87

Thom, C., Putman, M. E., Gibson, B. K., et al. 2006, ApJ, 638, L97

Thom, C., Peek, J. E. G., Putman, M. E., et al. 2008, ApJ, 684, 364

Wakker, B. P., \& van Woerden, H. 1998, ARA\&A, 35, 217

Wakker, B. P., York, D. G., Howk, J. C., et al. 2007, ApJ, 670, L113

Wakker, B. P., York, D. G., Wilhelm, R., et al. 2008, ApJ, 672, 298

Wannier, P., \& Wrixon, G. T. 1972, ApJ, 173, L119

Welty, D. E., Frisch, P. C., Sonneborn, G., \& York, D.G 1999, ApJ, 512, 636

Werner, K., \& Rauch, T. 2015, A\&A, 584, A19

van Woerden, H., Schwarz, U. J., Peletier, R. F., Wakker, B. P., \& Kalberla, P. M. W. 1999, Nature, 400, 138 